\title{
模形式空间关于 Hecke 算子的对角化
}

\section{王学理}

(广州师范学院数学系.广州 510400)

\section{关键词模形式空间、Hecke 算子、对角化、新形}

Atkin 和 Lehner ${ }^{[1]}$ 研究了权为 $2 k$ 的群 $\Gamma_{0}(N)$ 的歧点形式空间 $S_{2 k}(N)$ 的新形式 (newforms) 理论, 证明了 $S_{2 k}(N)=S_{2 k}^{\text {new }}(N) \oplus S_{2 k}^{\text {old }}(N)$, 其中的 $S_{2 k}^{\text {new }}(N)$ 有一组由所有 Hecke 算子 的特征向量构成的基, 而 $S_{2 k}^{\mathrm{old}}(N)$ 则只有一组关于 Hecke 算子 $T(p)((p, N)=1)$ 的公共特征向 量构成的基. Manickam, Ramakrishnan 和 Vasudevan ${ }^{[2,3]}$ 研究权为 $k+1 / 2$ 的新形式理论, 讨论了空间 $S_{2 k}(q)$ 关于所有的 Hecke 算子的对角化, 其中 $q \equiv 3(4)$ 是一个素数.

在本文中, 我们将要研究空间 $M_{2 k}(q)$ 及 $M_{k+1 / 2}(q)$ 关于所有 Hecke 算子的对角化. 此处 $q$ $\equiv 3(4)$ 是一个素数, $k \geqslant 2$ 是一个正整数.

\section{1 记 号}

设 $k \geqslant 2$ 是一个自然数, $q$ 是一个素数, $q \equiv 3(4)$. 设 $p$ 是任意一个不等于 $q$ 的素数, $f \in M_{2 k}$ $(q)$ 是一个权为 $2 k$ 的全纯模形式. 设 $f=\sum_{n=0}^{\infty} \hat{f}(n) e(n z)$ 是其 Fourier 展开式, 其中 $e(x)=\exp$ (2rix). 记 $T(p)$ 和 $U(q)$ 是 $M_{2 k}(q)$ 上的 Hecke 算子, 有

$$
\begin{aligned}
& f \mid T(p)=\sum_{n=0}^{\infty}\left(\hat{f}(n p)+\mathrm{p}^{2 k-1} \hat{f}(n / p)\right) e(n z), \\
& f \mid U(q)=\sum_{n=0}^{\infty} \hat{f}(q n) e(n z),
\end{aligned}
$$

其中 $\hat{f}(n / p)=0$ 如果 $p \nmid n$. 又设 $g \in M_{k+1 / 2}(q, \chi)$ 是一个权为 $k+1 / 2$ 的全纯半整权模形式, $g=\sum_{n=0}^{\infty} \hat{f}(n) e(n z)$ 是其 Fourier 展开式. 记 $T\left(p^{2}\right)$ 和 $U\left(q^{2}\right)$ 是 $M_{k+1 / 2}(q, \chi)$ 的 Hecke 算子 ( $\chi$ 是模 $q$ 的一个二次特征), 有

$$
\begin{aligned}
& g \mid T\left(p^{2}\right)=\sum_{n=0}^{\infty}\left(\hat{g}\left(p^{2} n\right)+\chi(p)\left(\frac{(-1)^{k} n}{p}\right) p^{k-1} \hat{g}(n)+\chi\left(p^{2}\right) P^{2 k-1} \hat{g}\left(n / p^{2}\right)\right) e(n z), \\
& g \mid U\left(q^{2}\right)=\sum_{n=0}^{\infty} \hat{g}\left(q^{2} n\right) e(n z),
\end{aligned}
$$

其中 $\hat{g}\left(n / p^{2}\right)=0$ 如果 $p^{2} \nmid n$.

1993-05-17 收稿. 


\section{$2 \mathbf{M}_{2 k}(q)$ 和 $M_{k+\frac{1}{2}}(q, \chi)$ 的对角化}

我们知道空间 $M_{2 k}(q)$ 和 $M_{k+\frac{1}{2}}(q, \chi)$ 可以分解如下:

$$
\begin{aligned}
& M_{2 k}(q)=E_{2 k}(q) \oplus S_{2 k}(q)=E_{2 k}(q) \oplus S_{2 k}^{\text {new }}(q) \oplus S_{2 k}(1) \oplus S_{2 k}(1) \mid U(q), \\
& M_{k+\frac{1}{2}}(q)=E_{k+\frac{1}{2}}(q, \chi) \oplus S_{k+\frac{1}{2}}(q, \chi)=E_{k+\frac{1}{2}}(q, \chi) \oplus S_{k+\frac{1}{2}}^{\text {new }}(q) \oplus S_{k+\frac{1}{2}}(1) \oplus S_{k+\frac{1}{2}}(1) \mid U\left(q^{2}\right) \text {. }
\end{aligned}
$$

现在我们考虑两个空间 $S_{2 k}(q)$ 和 $S_{k+\frac{1}{2}}(q, \chi)$. 由文献[3], 空间 $S_{2 k}(q)$ 关于所有 Hecke 算 子 $T(p)$ 和 $U(q)$ 可对角化. 事实上, 由文献[3] 中定理有

$$
S_{2 k}(q)=\underset{i=1}{\oplus} \mathbb{C}\left(f_{i}(z)\right), s=\operatorname{dim} S_{2 k}(q),
$$

其中每一个 $f_{i}$ 都是所有 Hecke 算子的公共特征向量且

$$
\begin{aligned}
& f_{i} \mid T(p)=\hat{f}_{i}(p) f_{i}, \\
& f_{i} \mid U(q)=\hat{f}_{i}(q) f_{i}, \\
& \hat{f}_{i}(1)=1 .
\end{aligned}
$$

由文献[2] 定理 7, 存在一个从空间 $S_{k+\frac{1}{2}}(q, \chi)$ 到 $S_{2 k}(q)$ 的同构映射 $\varphi_{k, q, \chi}$, 它是一些 Shimura 提升的线性组合, 使得

且

$$
\begin{gathered}
T\left(P^{2}\right) \varphi_{k, q, \chi}=\varphi_{k, q, \chi} T(p), \\
U\left(q^{2}\right) \varphi_{k, q, \chi}=\varphi_{k, q, \chi} U(q) . \\
\bar{f}_{i}=\varphi_{k, q, \chi}^{-1}\left(f_{i}\right),
\end{gathered}
$$

则易知

所以我们有

$$
\begin{aligned}
& \bar{f}_{i} \mid T\left(P^{2}\right)=\hat{f}_{i}(P) \bar{f}_{i}, \\
& \bar{f}_{i} \mid U\left(q^{2}\right)=\hat{f}_{i}(q) \bar{f}_{i} .
\end{aligned}
$$

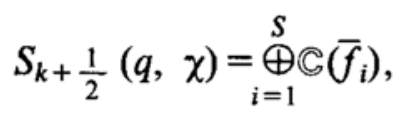

其中每一个 $\bar{f}_{i}$ 是所有 Hecke 算子的公共特征向量.

现在, 由文献[4] 可知空间 $E_{k+\frac{1}{2}}(q, \chi)$ 中存在一组基, 它们是所有 Hecke 算子的公共特 征向量, 从而我们知道在 $M_{k+\frac{1}{2}}(q, \chi)=E_{k+\frac{1}{2}}(q, \chi) \oplus S_{k+\frac{1}{2}}(q, \chi)$ 中存在一组由所有 Hecke 算子的公共特征向量构成的基, 即 $M_{k+\frac{1}{2}}(q, \chi)$ 关于所有 Hecke 算子可对角化.

同构映射 $\varphi_{k, q, \chi}: S_{k+\frac{1}{2}}(q, \chi) \rightarrow S_{2 k}(q)$ 是一些 Shimura 提升的线性组合, 所以 $\varphi_{k, q, \chi}$ 的 定义可以扩展到整个空间 $M_{k+\frac{1}{2}}(q, \chi)$ 上 ${ }^{[4]}$. 事实上, 这样扩展后的 $\varphi_{k, q, \chi}$ 是 $M_{k+\frac{1}{2}}^{+}(q, \chi)$ 到 $M_{2 k}(q)$ 上的一个同构 ${ }^{[5]}$, 这里 $M_{k+\frac{1}{2}}^{+}(q, \chi)$ 是 Kohnen ${ }^{[6]}$ 引进的 “+ 空间”, 定义如下:

其中

$$
M_{k+\frac{1}{2}}^{+}(q, \chi)=E_{k+\frac{1}{2}}^{+}(q, \chi) \oplus S_{k+\frac{1}{2}}(q, \chi),
$$

$$
E_{k+\frac{1}{2}}^{+}(q, \chi)=\left\{f=\sum_{n \geqslant 0} a(n) e(n z) \in E_{k+\frac{1}{2}}(q, \chi) \mid a(n)=0, \text { 如果 }(-1)^{k} \ln \equiv 2,3(\bmod 4)\right\} .
$$


此处 $l$ 是 $N=q$ 的一个因子,使得

$$
\chi(d)=\left(\frac{l}{d}\right),(d, 2 q)=1 .
$$

利用文献[4] 中的结果, 我们不难证明

$$
\begin{aligned}
& g_{1}=g_{\mathrm{k}+\frac{1}{2}}(l, 1,4 q)(z)-g_{k+\frac{1}{2}}(l, 1,8 q)(z), \\
& g_{2}=g_{k+\frac{1}{2}}(l, q, 4 q)(z)-g_{k+\frac{1}{2}}(l, q, 8 q)(z)
\end{aligned}
$$

是 $E_{k+\frac{1}{2}}^{+}(q, \chi)$ 的一组基, 并且 $g_{1}, g_{2}$ 是所有 Hecke 算子的公共特征向量, 其中 $g_{k+\frac{1}{2}}(l, m$, $4 q), g_{k+\frac{1}{2}}(l, m, 8 q)(m=1, q)$ 如文献[4] 中所定义. 于是由

$$
\begin{aligned}
& \varphi_{k, q, \chi}\left(E_{k+\frac{1}{2}}^{+}(q, \chi)\right)=E_{2 k}(q), \\
& \varphi_{k, q, \chi} T(p)=T\left(p^{2}\right) \varphi_{k, q, \chi}, \\
& \varphi_{k, q, \chi} U(q)=U\left(q^{2}\right) \varphi_{k, q, \chi},
\end{aligned}
$$

可知 $\varphi_{k, q, \chi}\left(g_{1}\right), \varphi_{k, q, \chi}\left(g_{2}\right)$ 是 $E_{2 k}(q)$ 的一组基, 且它们是所有 Hecke 算子的公共特征向量. 从而可知 $M_{2 k}(q)=E_{2 k}(q) \oplus S_{2 k}(q)$ 关于所有 Hecke 算子可对角化.

我们总结上面的结果如下:

定理 设 $q \equiv 3(\bmod 4)$ 是一个素数, 则有以下的直和分解:

$$
\begin{gathered}
M_{2 k}(q)=\mathbb{C} g_{1} \oplus \mathbb{C} g_{2} \oplus\left(\stackrel{s}{\oplus} \underset{i}{G} f_{i}\right), \\
M_{k+\frac{1}{2}}(q, \chi)=\mathbb{C} g_{k+\frac{1}{2}}(l, 4,4 q) \oplus \mathbb{C} g_{k+\frac{1}{2}}(l, 1,4 q) \oplus \mathbb{C} g_{k+\frac{1}{2}}(l, 4 q, 4 q) \\
\oplus \mathbb{C} g_{k+\frac{1}{2}}(l, q, 4 q) \oplus\left(\stackrel{s}{\left.\oplus \mathbb{C} \bar{f}_{i}\right)},\right.
\end{gathered}
$$

其中 $s=\operatorname{dim} S_{2 k}(q), f_{i}$ 和 $\bar{f}_{i}$ 如 (1) 和 (2) 式所示. $g_{1}$ 和 $g_{2}$ 如 (3) 式所示, 而 $g_{k+\frac{1}{2}}(l, j, 4 q)(j=1$, $4, q, 4 q)$ 如文献 [4] 中所示. 每一个上述一维子空间都是所有 Hecke 算子的公共特征空间.

\section{参考 文 䵧}

[1] Atkin, A. O. L., Lehner, J., Math. Ann., 1970, 185:134- 160.

[2] Manickam, M., Ramakrishnan, B., Vasudevan, T. C., J. Number Theory, 1990, 34:201-224.

[ 3] Manickam, M., Ramakrishnan, B., Vasudevan, T. C., J. of Number Theory, 1992, 40:251-253.

[4] 裴定一,数学学报, 1987, 30(4):512- 522 .

[ 5] Manickam, M., Ramakrishnan, B., Vasudevan, T. C., J. of Number Theory, 1992, 42:369-371.

[6] Kohnen, W., J. Reine Angew. Math., 1982, 333:32-72. 